\title{
The Position of the Fourth Segment of Domain 4 Determines Status of the Inactivation Gate in $\mathrm{Na}^{+}$Channels
}

\author{
Ya-Chin Yang ${ }^{1}$ and Chung-Chin Kuo ${ }^{1,2}$ \\ ${ }^{1}$ Department of Physiology, National Taiwan University College of Medicine, and ${ }^{2}$ Department of Neurology, National Taiwan University Hospital, Taipei \\ 100, Taiwan
}

\begin{abstract}
The fourth segment of domain 4 (S4/D4) in $\mathrm{Na}^{+}$channels is a voltage sensor especially implicated in channel inactivation. Although there has been evidence that S4/D4 moves externally during membrane depolarization, whether (and if so, how) the movement leads to conformational changes of the inactivation gate remains unknown. We added a positive charge just external to the outermost charged residue in S4/D4 by point mutations of residue F1625 (i.e., F1625R and F1625K). The inactivation curves as well as the kinetics of recovery from inactivation in these mutant channels are split into two components, one happening with and the other without channel activation/ deactivation. This is as if the "extra" positive charge induces new intermediate positions of S4/D4 and consequently new intermediate inactivation states uncoupled from channel activation/deactivation. The qualitatively similar but quantitatively very different findings between the F1625R and F1625K mutants further suggest a significantly different effect on the inactivation gate by a slight difference in the localization of the positive charge. On the other hand, neutral mutations of residue F1625 do not induce new inactivation states but shift the voltage dependence of different inactivation parameters in the voltage axis, as if only the relative tendency of S4/D4 to stay in its original outermost and innermost positions is altered. We conclude that S4/D4 movement not only decisively but also delicately controls the inactivation gate. Electrostatic interaction between the top charges of S4/D4 and the corresponding countercharges may play an essential role in the determination of S4/D4 position and therefore the inactivation status of the $\mathrm{Na}^{+}$channel.
\end{abstract}

Key words: activation-inactivation coupling; charge movement; inactivation; sodium channels; voltage sensors; gating charges

\section{Introduction}

In response to membrane potential changes, voltage-gated $\mathrm{Na}^{+}$ channels undergo a series of gating conformational changes, including activation and inactivation that concert to ensure voltage- and time-limited access to the pore and therefore appropriate shape and frequency of cellular discharges. For voltagedependent control of the gating machinery, a voltage-sensing device must be present in the channel protein. The fourth transmembrane segment (S4) in each of the four domains of the $\mathrm{Na}^{+}$ channel contains regularly spaced, positively charged amino acids and has been considered to be the primary voltage sensor of the channel (Catterall, 1986; Stühmer et al., 1989; Auld et al., 1990; Fleig et al., 1994; Kontis et al., 1997). The S4 segment in domain 4 (S4/D4) seems to be especially related to $\mathrm{Na}^{+}$channel inactivation, chiefly because neutralization of the positive charges in S4/D4 rather than in the other S4 segments would reduce gating charges coupled to the inactivation process (Stühmer et al., 1989; Chahine et al., 1994; Chen et al., 1996; Kühn and Greeff, 1999; Sheets et al., 1999).

S4/D4 has been shown to move outward in response to membrane depolarization (Chahine et al., 1994; Yang and Horn, 1995; Yang et al., 1996, 1997). Although the voltage-dependent movement of S4/D4 is consistent with what one would imagine for a voltage sensor, the functional significance of such a movement has never been established (Horn, 2000). It remains unclear whether (and if so, how) the movement of S4/D4 is coupled to the conformational changes in the inactivation gate. Could S4/D4

Received 0ct. 29, 2002; revised April 1, 2003; accepted April 3, 2003

This work is supported by Grant NHRI-EX91-9105NN from the National Institute of Health, Taiwan.

Correspondence should be addressed to Chung-Chin Kuo, Department of Physiology, National Taiwan University College of Medicine, No. 1, Jen-Ai Road, First Section, Taipei 100, Taiwan. E-mail: cckuo@ha.mc.ntu.edu.tw.

Copyright $\odot 2003$ Society for Neuroscience $\quad$ 0270-6474/03/234922-09\$15.00/0 movement (S4/D4 position) control the extent of inactivation? If so, how much S4/D4 movement is significant enough to "induce" inactivation? Also, because inactivation is strongly coupled to activation in $\mathrm{Na}^{+}$channels, are the other conformational changes (such as those related to channel activation) prerequisites for S4/D4 movement and channel inactivation? Answers to these questions should provide not only more conclusive evidence that S4/D4 is the voltage sensor responsible for $\mathrm{Na}^{+}$channel inactivation but also more insight into the causal relation between charge movements and gating conformational changes in the voltage-gated channels.

We made positively charged and other substitutions for F1625 (phenylalanine 1625), a residue located just external to the outermost charged residue (R1626 or "R1") of S4/D4 in the rat brain type IIA (rBIIA) $\mathrm{Na}^{+}$channel. $\mathrm{R} 1$ has been reported to emerge outside the membrane during depolarization and reach a negatively charged hydrophilic crevice (Chahine et al., 1994; Yang and Horn, 1995; Yang et al., 1997). This is similar to the case of the shaker $\mathrm{K}^{+}$channel, in which the outermost charged residue of S4 moves close to a "countercharge" during activation (Elinder and Århem, 1999; Larsson and Elinder, 2000; Elinder et al., 2001a,b). Our results indicate that the added positive charge (at residue 1625) external to R1 may also have a chance to bind to the putative countercharges and consequently secure S4/D4 in an intermediate position to induce correlative gating changes. Moreover, a small S4/D4 movement itself seems to induce significant changes in the inactivation gate, regardless of channel activation or deactivation.

\section{Materials and Methods}

Molecular biology and expression of $\mathrm{Na}^{+}$channels. The plasmid pNa200 contains cDNA encoding the rBIIA $\mathrm{Na}^{+}$channel $\alpha$ subunit and the Xenopus- $\beta$-globin untranslated sequence (a kind gift from Dr. Alan L. 
Goldin, Department of Microbiology and Molecular Genetics, University of California, Irvine, CA). The whole coding region of pNa200 may be divided into two subfragments: XhoI-BglII and BglII-NotI fragments, each $\sim 3-4 \mathrm{~kb}$ in size. To obtain the parent construct suitable for mutagenesis, we subcloned the BglII-NotI subfragment into another small and easily growing vector pBSTA (also kindly provided by Dr. Alan L. Goldin). Using this construct as a template, site-directed mutagenesis (with the QuikChange mutagenesis system, Stratagene, La Jolla, CA) was performed to obtain the F1625 mutations. The mutation-containing BglII-NotI fragment was then excised and transferred back into the pNa200 expression vector. Mutations were verified by DNA sequencing, and two independent clones for each mutant were examined to ensure the lack of any inadvertent mutations. The full-length cRNA transcript was then synthesized from pNa200 using T7 polymerase (the T7 mMESSAGE mMACHINE transcription kit, Ambion, Austin, TX) and injected into isolated follicle-free Xenopus oocytes (stages V-VI), which were maintained at $18^{\circ} \mathrm{C}$ for $1-7 \mathrm{~d}$ before electrophysiological recordings.

Electrophysiological recordings and data analysis. During recording, oocytes were put in a chamber continuously perfused with ND-96 solution containing (in mM): $96 \mathrm{NaCl}, 2 \mathrm{KCl}, 1 \mathrm{MgCl}_{2}, 1.8 \mathrm{CaCl}_{2}, 5 \mathrm{HEPES}, \mathrm{pH}=$ 7.6. Macroscopic $\mathrm{Na}^{+}$currents were recorded at room temperature $\left(\sim 25^{\circ} \mathrm{C}\right)$ by a standard two-microelectrode voltage-clamp amplifier with a virtual ground circuit (model OC-725C, Warner Instrument, Hamden, CT). Both voltage-sensing and current-passing electrodes were filled with $3 \mathrm{M} \mathrm{KCl}$ and had series resistance of $0.1-0.3 \mathrm{M} \Omega$. Immediately after the establishment of the two-electrode voltage-clamp configuration, the oocyte was clamped at $-140 \mathrm{mV}$ for at least $3 \mathrm{~min}$ to ensure recovery of $\mathrm{Na}^{+}$channels from inactivation. Data were acquired using the Digidata-1200 analog/digital interface with pCLAMP software (Axon Instruments, Foster City, CA). The sampling rates were $10-50 \mathrm{kHz}$, and all statistics in this study are given as mean \pm SE of mean.

To plot the inactivation curves, we measured the current at $+5 \mathrm{mV}$ after a $9 \mathrm{sec}$ prepulse at different voltages from a holding potential of $-140 \mathrm{mV}$. The available fraction is defined as the normalized peak current relative to the maximal current evoked and is plotted against the prepulse voltage. The relatively long inactivating pulses were made to ensure steady-state inactivation. This is because in some mutants part of the inactivation happens at very negative membrane potentials at which inactivation could proceed with only very slow kinetics. For example, comparison of the inactivation curves with prepulse durations of 500 msec and $9 \mathrm{sec}$ reveals that the positive-charge mutants (F1625K and F1625R) require a relatively long time to achieve steady-state inactivation (although the two-component nature of the inactivation curve remains clear with either prepulse duration) (Fig. 1). On the other hand, there is unlikely a significant contamination of the inactivation curve by additional slow inactivation with the 9-sec-long prepulse because of the close resemblance between the 500-msec-prepulse and the 9-sec-prepulse inactivation curves in either the wild-type or neutral mutant channels (only negligible shift within $5 \mathrm{mV}$ ) (Fig. 1). The inactivation curves can be reasonably fitted with a Boltzmann function $1 /\left(1+\exp \left(\left(V-V_{\mathrm{h}}\right) / k\right)\right)$ for the wild-type and neutral mutant channels, whereas summation of two Boltzmann functions $f_{1} /\left(1+\exp \left(\left(V-V_{\mathrm{h} 1}\right) / k_{1}\right)\right)+f_{2} /(1+\exp ((V-$ $\left.\left.V_{\mathrm{h} 2}\right) / k_{2}\right)$ ) is required for fitting the data from the positive-charge mutants, where $f_{1}$ and $f_{2}$ are the fractions of the first and second components of inactivation, respectively (see Results) $\left(f_{1}+f_{2}=1\right)$. $V$ denotes the prepulse potential in millivolts. $V_{\mathrm{h}}$ and $k$ have their usual meanings. For the normalized conductance-voltage relationship, the oocyte was first held at $-140 \mathrm{mV}$ and stepped to different test voltages to elicit $\mathrm{Na}^{+}$ currents. Because the maximal current usually appears at a test voltage around $-20 \mathrm{mV}$, we make a regression line of the data points between +10 and $+40 \mathrm{mV}$ in the current-voltage plot. The reversal potential $\left(V_{\text {rev }}\right)$ is estimated by extrapolating the regression line to the zero current level (the transverse axis), and the maximal peak conductance $\left(G_{\max }\right)$ is given by the slope of the line. The normalized conductance is defined as $I_{\text {peak }} /\left(\left(V-V_{\text {rev }}\right) \times G_{\text {max }}\right)$, where $I_{\text {peak }}$ and $V$ denote the peak current and the test voltage during the step depolarization, respectively. The normalized conductance is plotted against the test voltage and then fitted with a Boltzmann function $1 /\left(1+\exp \left(\left(V_{\mathrm{h}}-V\right) / k\right)\right)$.

For the measurement of the kinetics of recovery from inactivation, the oocyte was held at $-140 \mathrm{mV}$ and pulsed twice to $-10 \mathrm{mV}$ (each for 100

\section{- $500 \mathrm{~ms}$ prepulse}

- 9 s prepulse

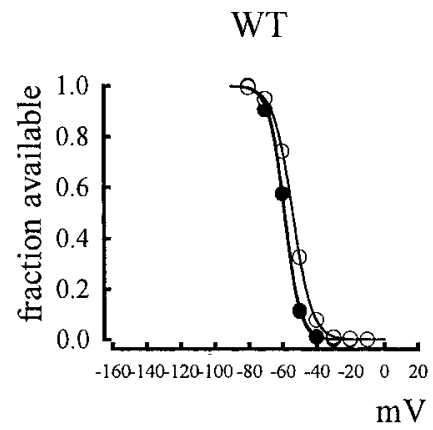

F1625Q

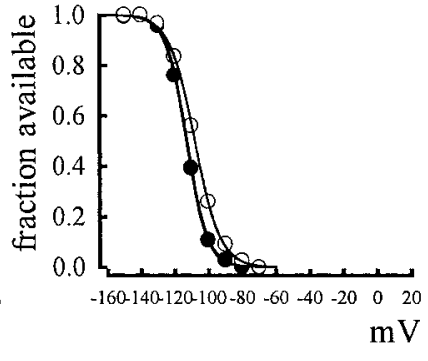

F1625K

F1625R
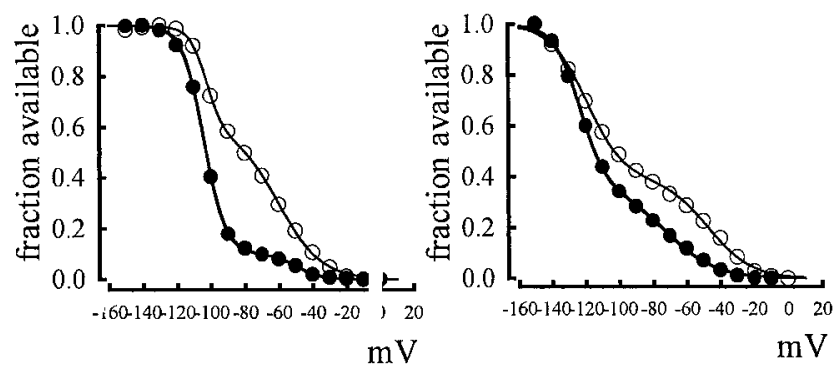

Figure 1. Comparison of the sample inactivation curves with prepulse durations of $500 \mathrm{msec}$ (O) and $9 \sec ()_{)}$in the same oocyte for each mutant or wild-type $\mathrm{Na}^{+}$channels. The inactivation curves ( 500 msec prepulse vs 9 sec prepulse) are fitted with single Boltzmann functions, with $V_{\mathrm{h}}$ values (in millivolts) of -54.0 ( $500 \mathrm{msec}$ prepulse) versus -58.8 ( 9 sec prepulse) and -107.9 ( $500 \mathrm{msec}$ ) versus -112.7 ( $9 \mathrm{sec})$, and $k$ values of $5.6(500 \mathrm{msec})$ versus $4.5(9 \mathrm{sec})$ and 7.4 ( $500 \mathrm{msec}$ ) versus 6.0 ( $9 \mathrm{sec}$ ) for the wild-type and the F16250 mutant, respectively. For the other two mutants, the inactivation curves are fitted with the summation of two Boltzmann functions, with $V_{\mathrm{h} 1}$ values (in millivolts) of -102.6 (500 msec) versus -104.1 ( $9 \mathrm{sec}$ ) and -119.0 ( $500 \mathrm{msec}$ ) versus $-123.3(9 \mathrm{sec}), V_{\mathrm{h} 2}$ values (in millivolts) of -60.4 ( $500 \mathrm{msec}$ ) versus -49.4 ( $9 \mathrm{sec})$ and -47.1 ( $500 \mathrm{msec})$ versus -71.8 ( $9 \mathrm{sec}), k_{1}$ values of 4.8 (500 $\left.\mathrm{msec}\right)$ versus $6.2(9 \mathrm{sec})$ and 10.9 ( $500 \mathrm{msec})$ versus $7.7(9 \mathrm{sec}), k_{2}$ values of $12.6(500 \mathrm{msec})$ versus $7.2(9 \mathrm{sec})$ and 12.7 ( $500 \mathrm{msec})$ versus 14.5 ( $9 \mathrm{sec})$, and $f_{1}$ values of $0.40(500 \mathrm{msec})$ versus $0.90(9 \mathrm{sec})$ and 0.61 ( $500 \mathrm{msec}$ ) versus 0.64 ( $9 \mathrm{sec}$ ) for the F1625K and F1625R mutants, respectively.

msec). Between the two pulses there was an intervening gap lengthened by $0.1 \mathrm{msec}$ (or longer time) at a given recovery potential. The extent of recovery, which is represented by the ratio between the peak currents in the second and first pulses, is plotted against the length of the gap to show the recovery time course of the channel. For the wild-type and neutral mutant channels, there is an initial delay followed by an exponential phase in the recovery courses. To exclude influences from the initial delay and any ultra-slow phase of recovery, we fitted the data points between 10 and $85 \%$ recovery with monoexponential function, which is extrapolated to the zero recovery level (the time axis) to define the length of the initial delay. Because the recovery courses immediately after the initial delay are approximately linear (see Fig. $5 A, B$ ), in the experiments examining the voltage dependence of the recovery rates (see Figs. $5 C, 7 A$ ), the recovery rates are determined by fitting the three data points (between the first two of which is the greatest increase in the extent of recovery right after the delay) with a linear regression function of the form: fraction recovered $=$ recovery rate $\times$ recovery duration (the length of the recovery gap). This simplified approach is to facilitate the measurement, especially at relatively positive recovery potentials where the recovery may be very slow and take a long time to reach $85 \%$ recovery. We have verified the validity of this simplified approach at more negative potentials, at which the result of the simplified measurement is very much consistent with the recovery rate given by the inverse of the time constant from the exponential fit to the recovery course (data not shown). In the cases of the 
positive-charge mutants in which there is no evident initial delay but a slow initial phase of recovery, we leave out the initial phase (roughly the initial $\sim 25 \%$ ) of recovery and fit the other data points with a monoexponential curve, which is extrapolated to the zero recovery level to define the "delay" of the second phase of recovery. The recovery rates of the second phase of recovery are then estimated with a linear regression function by the same way as those in the wild-type and neutral mutants.

\section{Results}

Positive-charge substitutions for F1625 facilitate inactivation with split of the steady-state inactivation curve into two components

Substitutions for F1625 with positively charged amino acids lysine (F1625K) and arginine (F1625R) dramatically shift the inactivation curves in the hyperpolarized direction, suggesting stabilization of the channel in the inactivated state at more hyperpolarized potentials (Fig. 2A). However, the overall voltage dependence of steady-state inactivation cannot be described satisfactorily by a single Boltzmann function. Instead, the curves are reasonably described by the summation of two Boltzmann functions. The "twocomponent" inactivation curve implies the existence of at least two different processes of inactivation in these F1625 positivecharge mutants. One of the inactivation processes (the first component of the inactivation curve) seems to reduce the available (resting) $\mathrm{Na}^{+}$channels at very hyperpolarized potentials. However, this process itself can only partly but not fully inactivate the channel, and the other process that becomes manifest at more depolarized potentials is required to achieve complete steadystate inactivation (the second component of the inactivation curve). It is plausible that the mutants with an added positive charge just external to the $\mathrm{R} 1$ residue may facilitate the movement of S4/D4 to an "intermediate" position by interacting with some of the putative countercharge(s) of S4/D4. Because S4/D4 is presumably a voltage sensor, its distribution from the resting to the intermediate position is likely a voltage-dependent process, accounting for the first component of the inactivation curve. Inactivation apparently cannot be complete even if S4/D4 is already in the intermediate (rather than in the "normal") inactivation position. In other words, the intermediate position seems to correspond to an unstable inactivated state in which the $\mathrm{Na}^{+}$ channel would still have a significant chance of being available. More depolarized potentials are necessary to initiate another voltage-dependent (Boltzmann) distribution, namely distribution of S4/D4 between the intermediate and a more external (the normal inactivation) position, which makes a stable inactivated state of the $\mathrm{Na}^{+}$channel with a negligible chance of being available. The preceding proposal contains an idea of coupling between the position of S4/D4 and the extent of inactivation. It is also notable that the F1625R mutant shows a more negatively shifted but smaller first component of the inactivation curve than the F1625K mutant. This finding may suggest that in the F1625R mutant there is a more easily achieved but less external intermediate position of S4/D4. Arginine might be more suitable than lysine to interact with the countercharge(s) originally responsible for stabilization of the outwardly translocated constitutive arginines in S4/D4 and therefore has a larger binding energy and consequently more negative shift of the
B

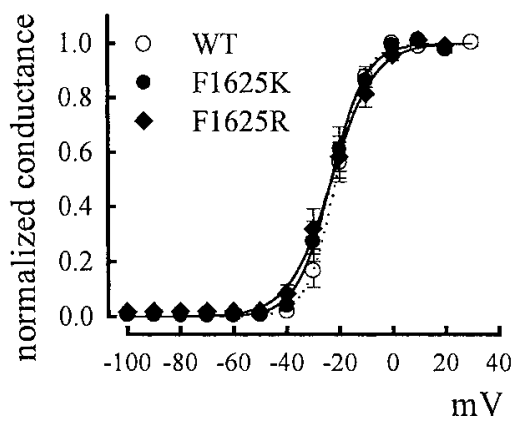

$\mathrm{mV}$

Figure 2. Steady-state inactivation and activation curves of F1625K and F1625R mutants. A, Steady-state inactivation curves $(n=4-7)$ are obtained by the protocol described in Materials and Methods. The lines are fits with a single Boltzmann function for the wild type (dotted line) or the summation of two Boltzmann functions for the F1625K and utants (solid lines). The $V_{\mathrm{h}}$ and $k$ values from the fits are $-64.2 \mathrm{mV}$ and 5.0, respectively, for the wild-type (he two mutant channels are shifted in the voltage axis and superimposed to demonstrate the existence unction with $V_{\mathrm{h}}$ values of $-21.4,-23.0$, and $-22.9 \mathrm{mV}$, and $k$ values of $5.2,6.5$, and 8.1 for the wild-type (dotted line), (solid lines), respectively.

curve. In other words, the differences in length $(\sim 1.3 \AA$ longer in arginine than in lysine) and other physicochemical properties (e.g., a plane resonating $\mathrm{H}_{2} \mathrm{~N}-\mathrm{C}=\mathrm{N}^{+}-\mathrm{H}_{2}$ structure in arginine but not in lysine) of the substituting side chains may account for the more significant interaction of F1625R (than F1625K) with the countercharge(s) of S4/D4 in the presence of less depolarized potentials and less external translocation of S4/D4. In contrast, the voltage range necessary to trigger the second distribution of S4/D4 to the normal inactivation position is similar in both mutants ( $V_{\mathrm{h} 2}-73.0 \mathrm{mV}$ vs $-69.2 \mathrm{mV}$ ) (Fig. $2 A$ ), suggesting a mutation-independent determinant for this second inactivation process.

\section{The F1625 positive-charge mutants do not change the} activation curve

Because inactivation is coupled to activation in $\mathrm{Na}^{+}$channels (Goldman and Schauf, 1972; Armstrong and Bezanilla, 1977; Bezanilla and Armstrong, 1977; Goldman and Kenyon, 1982), it is possible that the apparent effect of the mutations on inactivation actually results from alteration of channel activation. We therefore examined the activation curve in the mutant channels (Fig. 2 B). In sharp contrast to the cases of the inactivation curve, no significant changes in either the midpoint or the shape of the conductance-voltage curve are found with the positive-charge mutations of F1625. The lack of effect on channel activation strongly suggests that the inactivation process itself is altered by the mutations. In addition, if inactivation is affected but activation is not, then inactivation and activation is somewhat "uncoupled" in these mutant channels. In this regard, it is interesting that the voltage range of the second component of the inactivation curve in these positive-charge mutants $\left(V_{\mathrm{h} 2}\right.$ values are -69.2 and $-73 \mathrm{mV}$ for the $\mathrm{F} 1625 \mathrm{~K}$ and the F1625R mutants, respectively) (Fig. $2 \mathrm{~A}$ ) is similar to that of the inactivation curve in the wild-type channels $\left(V_{\mathrm{h}}\right.$ is $-64.2 \mathrm{mV}$ ). This may suggest that the second component of the inactivation curve in the mutants represents an inactivation process analogous to the inactivation in the wild-type channel and thus is coupled to (and facilitated by) the unchanged activation process. Under such circumstances, 
A

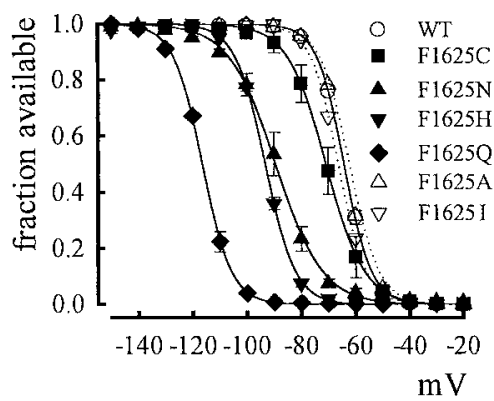

B

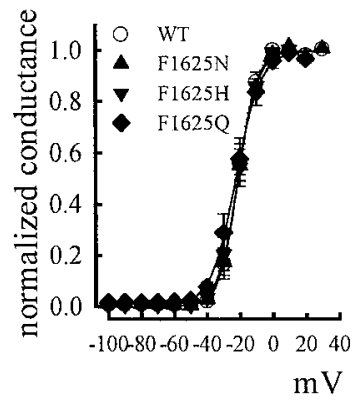

Figure 3. Inactivation and activation properties of $\mathrm{F} 1625$ neutral mutants. $A$, Steady-state inactivation curves of the neutral mutants ( $n=4-5$ for each mutant). The lines are fits with a Boltzmann function with $V_{h}$ values (in millivolts) of $-64.2,-63.8$, $-66.3,-70.9,-89.4,-93.3$, and -116.5 and $k$ values of $5.0,5.0,5.1,7.0,8.5,5.5$, and 5.3 for the wild type and the mutants with $\mathrm{A}, \mathrm{I}, \mathrm{C}, \mathrm{N}, \mathrm{H}$, and Q substitutions for F1625, respectively. The F1625A and F1625I mutants, in which the substituting side-chain groups cannot form hydrogen bonds, are illustrated with the dotted fitting lines, and the others are illustrated with the solid lines. $B$, Normalized conductance-voltage curves for several F1625 neutral mutants ( $n=4-9$ for each mutant). The $V_{\mathrm{h}}$ values from the fits with a Boltzmann function are $-21.4,-21.1,-21.2$, and $-22.5 \mathrm{mV}$, and the $k$ values are 5.2, 5.9, 6.1, and 7.7 for the wild-type, F1625N, F1625H, and F16250 mutant channels, respectively. C, The kinetics of macroscopic inactivation in the wildtype and neutral mutant channels. $\mathrm{Na}^{+}$currents are elicited by depolarization to $0 \mathrm{mV}$ from a holding potential of $-140 \mathrm{mV}$. The currents are normalized to the same peak amplitude and superimposed with one another. The dotted line indicates the zero current level.

the first component would possibly imply an inactivation process that happens without channel activation.

Neutral substitutions for F1625 facilitate inactivation without changes in the shape of the steady-state inactivation curve We have proposed that the effect of the positive-charge substitutions for F1625 on inactivation probably results from the interaction between the added positive charge and the putative countercharge(s) of S4/D4. To explore the necessity of a positive charge, we made neutral substitutions for F1625. Figure $3 A$ demonstrates that some neutral substitutions also shift the inactivation curve in the hyperpolarized direction. However, each set of data could be well described with a standard Boltzmann curve similar in shape to the inactivation curve of the wild-type channel. Under no circumstances could the neutral mutants produce the two-component inactivation curves like those found in the positive-charge mutants. In the neutral mutants, shift of the inactivation curve seems to happen only if the side-chain groups of the substituting amino acids are capable of forming hydrogen bonds. Moreover, the extent of the shift may be roughly correlated with the length of the side chain. For example, the glutamine substitution (F1625Q), which has the longest side chain among the neutral mutants, shows the most prominent shift of the inactivation curve. (Because of the qualitative similarity among the neutral mutants in the following experiments, we chose to show only the data from the three mutants F1625N, F1625H, and F1625Q.) On the other hand, the conductance-voltage curve is not significantly shifted by the neutral mutations (Fig. 3B), again indicating a direct effect of the mutations on inactivation. The F1625 neutral mutants thus seem to stabilize S4/D4 simply in the normal inactivation position (and thus stabilize exactly the same inactivation conformation as that in the wild-type channel) rather than create an additional intermediate position of S4/D4.

F1625 neutral mutants accelerate the kinetics of macroscopic inactivation

In addition to stabilization of the inactivated state, the F1625 neutral mutants also accelerate the kinetics of macroscopic inactivation (Fig. 3C). The decay phase after the rapid activation

$\mathrm{C}$

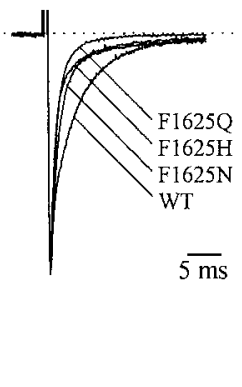

phase of the macroscopic $\mathrm{Na}^{+}$current has both a fast and a slow component. The biphasic current decay is typical for the rBIIA Na ${ }^{+}$channel $\alpha$ subunit expressed in Xenopus oocytes without coexpression of the $\beta 1$ subunit (Patton et al., 1994). The accelerated macroscopic inactivation in the F1625 neutral mutants appears ascribable to a significant decrease of the slow component of decay, and the order of acceleration of current decay could be roughly correlated with the order of shift of the inactivation curve in different mutants (Fig. 3A,C). The F1625Q mutant channels even show a macroscopic current decay well described by a monoexponential function (i.e., there is almost no slow component of decay), reminiscent of the macroscopic current of the wild-type channel in native neurons or in oocytes coexpressed with the $\beta 1$ subunit. These kinetic data are consistent with the view that neutral mutations at residue 1625 can stabilize the (fast) inactivated state and may also shed light on the mechanism of action of the $\beta 1$ subunit, the modulating effect of which on channel gating has been proposed to involve an extracellular loop in domain 4 (Qu et al., 1999).

\section{The F1625 neutral mutants display slower kinetics of recovery} from inactivation

In addition to the kinetics of development of macroscopic inactivation, we also compare the kinetics of recovery from inactivation among different neutral mutants. If the shift of the inactivation curves in the F1625 neutral mutants indeed results from stabilization of the inactivated conformation, these mutants probably should display slower kinetics in the recovery from inactivation at a given recovery potential. Figure $4 A$ shows the recovery course at a recovery potential of $-140 \mathrm{mV}$ in the wildtype channel, where there is an initial delay followed by an exponential phase (Kuo and Bean, 1994; Kuo and Yang, 2001). Qualitatively similar patterns of recovery courses are also noted in the F1625 neutral mutants (Fig. 4 B). The initial delay in the recovery course presumably results from silent deactivation of the inactivated $\mathrm{Na}^{+}$channel, which must first deactivate before it can recover from inactivation (Kuo and Bean, 1994). The initial delay thus could be viewed as the time it takes to deactivate the inactivated channel (i.e., the time required for a "quasi steady-state" distribution among different deactivated-inactivated states), and the subsequent exponential phase of recovery probably signals unbinding of the bound "hinged lid" from the deactivated-inactivated channels (i.e., reflecting a weighted average of the lid unbinding rates from different deactivated-inactivated states) (Kuo and Bean, 1994). The more stabilized inactivated state therefore would lead to longer initial delay and slower exponential recovery rate. For the neutral mutants examined, both the initial delay and the time constant of the subsequent exponential recovery phase increase with the larger shift of the inactivation curve (Fig. 4C,D). Such a positive correlation strongly supports a common mechanism underlying the changes in the inactivation curve, the initial delay, and the exponential phase of recovery in the F1625 neutral mutants, namely stabilization of the inactivated state of the channel. 
A
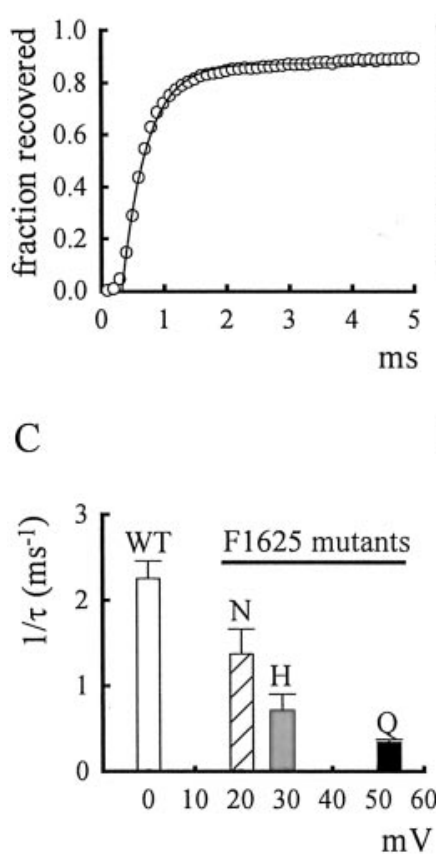

B

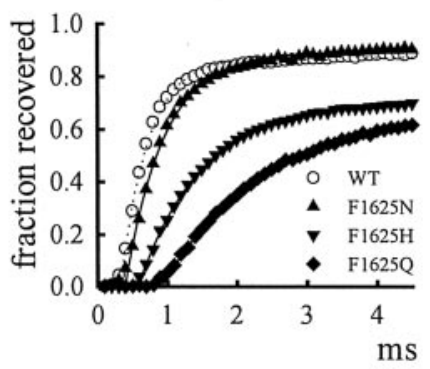

D

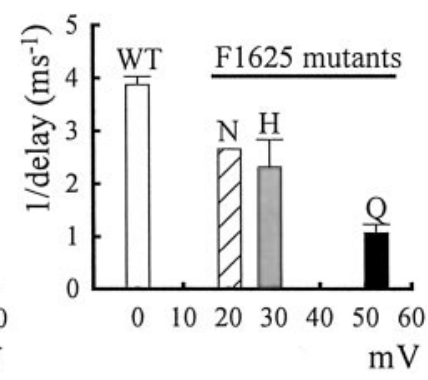

Figure 4. Recovery from inactivation at $-140 \mathrm{mV}$ in the wild-type and F1625 neutral mutant channels. $A$, The recovery time course in the wild-type channel. The line is a monoexponential function that is the best fit to the data points between 10 and $85 \%$ recovery. This exponential fit is extrapolated to the zero recovery level to define the length of the initial delay. $B$, The same experiments as in $A$ in F1625 neutral mutant channels. Note the lengthened initial delay as well as the correlative slowing of the subsequent exponential recovery course in the mutant channels. $C, D$, The inverse of the time constant $(\tau)$ of the exponential recovery course and the inverse of the initial delay from the experiments in $B(n=4-9)$ are plotted against the shift of the inactivation curve (the difference of $V_{\mathrm{h}}$ values between each mutant and the wildtype channels in millivolts). Both the inverse of the recovery time constant and the inverse of the initial delay are positively correlated with the shift of the inactivation curve.

The F1625Q mutant shifts the voltage dependence of recovery kinetics roughly to the same extent as the shift of the inactivation curve

We further examine the kinetics of recovery from inactivation over a wide range of recovery potentials in the F1625Q mutant (the neutral mutant with the greatest stabilization of inactivation). The F1625Q and the wild-type channels display very similar patterns of the voltage-dependent initial delay and the voltage-dependent subsequent exponential phase, except that the F1625Q mutant evidently requires more negative potentials to achieve the same recovery kinetics (Fig. $5 A, B$ ). Figure $5 C$ plots the recovery rates against the recovery potentials. The data can be simplistically approximated by a Boltzmann distribution on the basis of a quasi steady-state recovery model, in which the apparent recovery rate is dependent on the voltage-dependent distribution of the channel among the inactivated-deactivated states (Kuo and Bean, 1994; Kuo and Yang, 2001). The voltage dependence of the recovery rates in the F1625Q mutant channel shows a steepness similar to that in the wild-type channel (slope factor $k$ of $\sim 21.8$ and $\sim 17.8$, respectively) but is shifted in the hyperpolarized direction by $\sim 43 \mathrm{mV}$ (Fig. $5 C$ ). This is reminiscent of the $\sim 52 \mathrm{mV}$ midpoint difference of the inactivation curves between them (Fig. 3A). Also, the voltage dependence of the initial delay is very similar in steepness between the F1625Q and the wild-type channels (e-fold shortening per $\sim 70$ and $\sim 64 \mathrm{mV}$ hyperpolarization, respectively) but is again shifted by $\sim 50 \mathrm{mV}$ in the hyperpolarized direction in the F1625Q mutant (Fig. 5D). All of

A

B

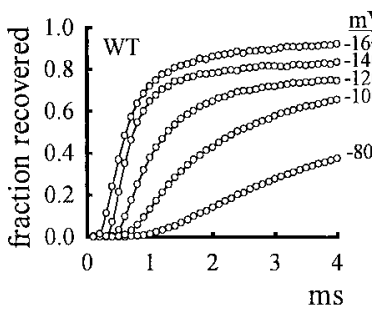

C

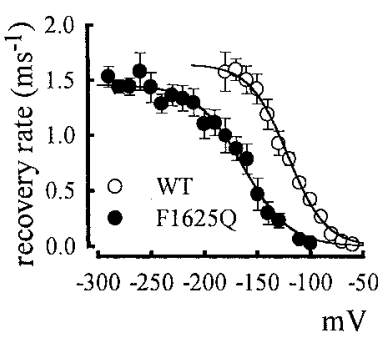

D

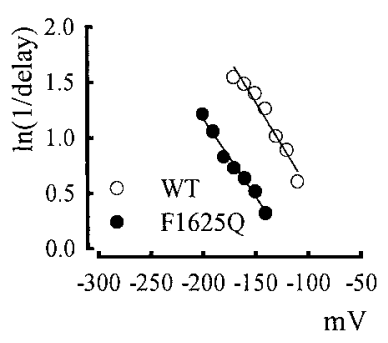

Figure 5. Kinetics of recovery from inactivation of wild-type and F1625Q channels over a wide range of recovery potentials. $A, B$, The kinetics of recovery from inactivation at different recovery potentials in the wild-type and $\mathrm{F} 1625 \mathrm{Q}$ mutant channels. The recovery (gap) potential is given beside each curve. The lines are drawn by hand and have no theoretical meanings. C, The voltage dependence of the recovery rates. The recovery rate is determined by the linear regression fitting method (see Materials and Methods for details; $n=7-11$ ). The recovery rate is plotted against the recovery potential and fitted by a standard Boltzmann function (solid lines) of the form: recovery rate $=R_{\text {satu }} /\left(1+\exp \left(\left(V-V_{\mathrm{h}}\right) / k\right)\right)$. The $V_{\mathrm{h}}$ values (in millivolts) are -121.4 and -164.0 , and the $k$ (the slope factor) values are 17.8 and 21.8 for the wild-type and $\mathrm{F} 16250$ channels, respectively. The $R_{\text {satu }}$ denotes the saturating recovery rate at extremely negative potentials, and the values are 1.6 and 1.5 (msec) $^{-1}$ for the wild-type and F16250 channels, respectively. $D$, The natural logarithm of the mean value of the inverse of the initial delay (in milliseconds) is plotted against the recovery potential ( $n=3-7$; the SE values are in general $<15 \%$ of the mean and are not shown). The lines are linear regression fits of the forms: $\ln (1 /$ delay $)=-1.0-0.016 \mathrm{~V}$, and $\ln (1 /$ delay $)=-1.7-0.014 \mathrm{~V}(\mathrm{~V}$ denotes the recovery potentials in millivolts) for the wild-type and F16250 mutant channels, respectively. The two lines have almost identical slope but are shifted in the voltage axis by $\sim 50 \mathrm{mV}$.

these quantitative data concerning different molecular events directly related to inactivation consistently indicate a similar steepness of voltage dependence but a $\sim 50 \mathrm{mV}$ shift in the voltage axis between the F1625Q and wild-type channels. This correlative shift in the voltage dependence of different inactivation parameters is also noted in the other neutral mutants, although the absolute value of the shift is different in different mutants (data not shown). These findings further strengthen the proposal that the inactivated conformation of the channel is stabilized to a different extent by different F1625 neutral mutations from a quantitative point of view.

The F1625 positive-charge mutants show no initial delay but a very slow initial phase of recovery from inactivation

We have seen that the steady-state inactivation curves in the positive-charge mutants are split into two components that are distinct from those in the wild-type and neutral mutant channels. The kinetics of recovery from inactivation in the positive-charge mutants are also very characteristic. The initial delay is no longer discernible in the F1625K and F1625R mutants (Fig. 6A), and the recovery course also is split into two components, i.e., an initial slow phase followed by an accelerated phase of recovery, both of which are voltage-dependent processes (Fig. $6 B, C$ ). For all of the recovery potentials that we tested $(-80$ to $-310 \mathrm{mV})$, there was 
A

WT

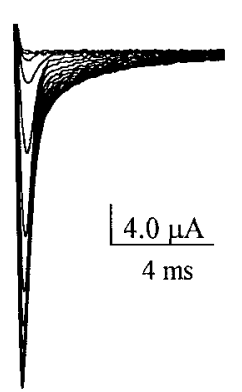

$\mathrm{B}$

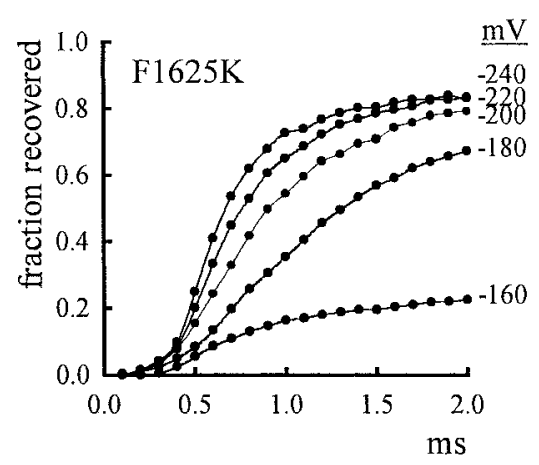

D

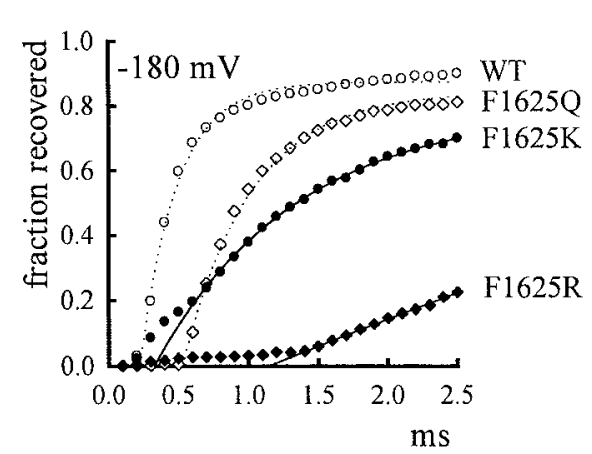

F1625K
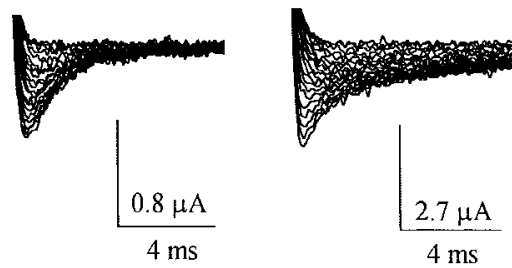

$\mathrm{C}$

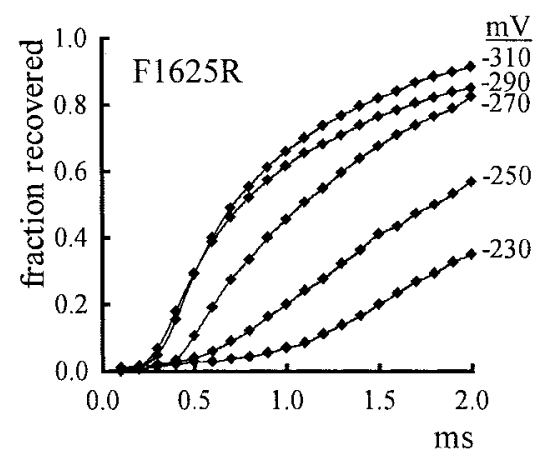

E

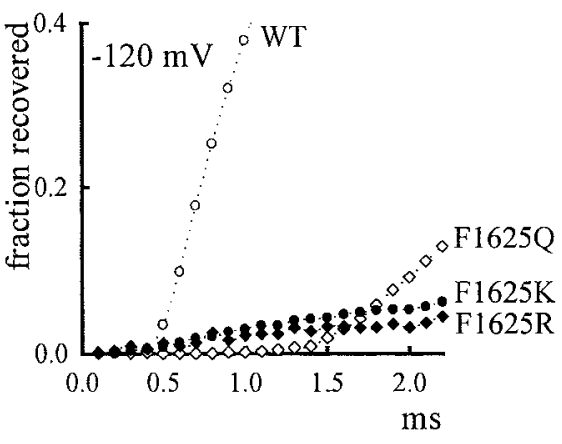

Figure 6. Lack of initial delay in the recovery courses of F1625K and F1625R mutant channels over a wide range of recovery potentials. $A$, The recovery potential at the intervening gap is $-140 \mathrm{mV}$. The gap is lengthened by $0.1 \mathrm{msec}$, and the recovering currents in the first 18-20 sweeps are shown. The growth of the currents in the wild-type and F1625Q mutant channels obviously has an initial delay that is manifested as the lack of current increment in the first few sweeps. This is especially so in the F16250 mutant, which has a much longer initial delay than the wild-type channel. In sharp contrast, there is no initial delay in the recovery course of the F1625K and F1625R mutant channels, and the currents in the second pulse start to increase after the first sweep. The vertical bar shows the current amplitude for $10 \%$ recovery in each individual oocyte. $B, C$, The time courses of recovery from inactivation at different recovery potentials in F1625K and F1625R mutant channels. The lack of initial delay is evident in both mutants over a wide range of recovery potentials. The lines are drawn by hand and have no theoretical meanings. $D$, The recovery time courses at a recovery potential of $-180 \mathrm{mV}$ in the wild-type, F1625Q, F1625K, and F1625R mutant channels (the data of F1625Q are taken from Fig. 5B). The dotted and solid lines are the monoexponential fits. $E$, A closer examination of the initial recovery course at a less hyperpolarized recovery potential $(-120 \mathrm{mV})$. The lines are drawn by hand and have no theoretical meanings. In sharp contrast to the evident delay in the wild-type and the F16250 mutant channels, there is no initial delay in the F1625K and F1625R mutant channels.

no evident initial delay observed in the F1625K and F1625R mutants. This is in sharp contrast to the evident voltage-dependent initial delay in the wild-type as well as neutral mutant channels (Fig. 6D,E). As we have noted, the initial delay is a manifestation of the coupling between deactivation and recovery from inacti- vation. The inactivated wild-type and neutral mutant $\mathrm{Na}^{+}$channels thus must be deactivated before they could recover from inactivation. In this regard, the "lack of delay" of the positive-charge mutant channels at all tested recovery potentials would indicate that in these inactivated mutant $\mathrm{Na}^{+}$channels deactivation is no longer an absolute prerequisite for the recovery from inactivation. Analogous to the two-component inactivation curve in these positive-charge mutants, the initial slow recovery phase or the lack of initial delay may be ascribable to the existence of an additional intermediate inactivated state that is uncoupled from channel deactivation. Deactivation, however, probably could still speed up the recovery process (facilitate unbinding of the bound hinged lid), as suggested by the following accelerated phase of recovery.

The rates of recovery from inactivation saturate at the same level in all of the mutant and wild-type channels

Figure $7 \mathrm{~A}$ demonstrates that the rates of the second phase of recovery are slower in the positive-charge mutants than in the neutral mutants (e.g., F1625Q) at each recovery potential, suggesting retardation of recovery from inactivation by the positivecharge mutations (especially F1625R). It is also evident that the voltage dependence of recovery rates in the F1625R mutant is much shallower than those in the wild-type and neutral mutant channels (Fig. 7A). These intriguing features of recovery from inactivation in the positive-charge mutants are consistent with the existence of an additional intermediate position of S4/D4 during its backward movement (and therefore an additional intermediate inactivated state during recovery from inactivation). It is also very interesting that the rates of recovery from inactivation saturate at very negative potentials and reach essentially the same value in all of the mutant (such as F1625N, $-\mathrm{H},-\mathrm{Q},-\mathrm{K},-\mathrm{R}$ ) and wild-type channels (Fig. $7 A$ ). This finding would strongly suggest that the bound hinged lid does have the same dissociation rate from the fully deactivated channel, no matter what changes or whether changes have been made to F1625. Residue 1625 thus does not seem to be located directly at the inactivation gate (the hinged lid and its receptor) of the $\mathrm{Na}^{+}$channel. Figure $7 B$ shows that the macroscopic current decay is also accelerated in the positive-charge mutant channels. We have noted that the acceleration of current decay in the neutral mutants could be correlated with the shift of the inactivation curve as well as the length of the substituting side chain (Fig. 3C); however, this trend is no longer kept in the positive-charge mutants. The F1625R mutant channel 


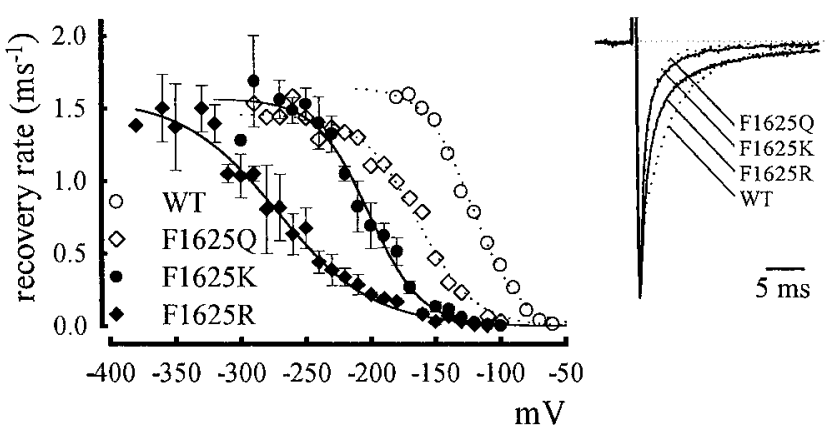

Figure 7. Kinetics of recovery from and development of inactivation in positive-charge mutants. $A$, Rates of recovery from inactivation at different recovery potentials in the wild-type and F1625 mutant channels. The recovery rates of the F1625K and F1625R mutants $(n=4-7)$ are estimated from the second, accelerated phase of recovery. The data points of the wild-type and F16250 mutant channels are taken from Figure 5C (error bars are omitted here for simplicity). The lines are the fits with a Boltzmann function, recovery rate $=R_{\text {satu }} /\left(1+\exp \left(\left(V-V_{\mathrm{h}}\right) / k\right)\right)$. The $V_{\mathrm{h}}$ values (in millivolts) are $-121.4,-164.0,-201.7$, and -271.6 , the $k$ values are 17.8 , $21.8,20.3$, and 36.4 , and the $R_{\text {satu }}$ values [in (millisecond) ${ }^{-1}$ ] are 1.6, 1.5, 1.6, and 1.6 for the wild-type, F1625Q, F1625K, and F1625R channels, respectively. Note the much shallower slope as well as the large negative shift in the F1625R mutant channel but the essentially identical saturating rates at very negative potentials for the wild-type and all mutant channels. $B$, The kinetics of macroscopic inactivation in the positive-charge mutants. The experimental protocol and the plot are the same as in Figure 3C. The sweeps of the wild-type and the $\mathrm{F} 1625 \mathrm{Q}$ mutant channels are taken from Figure 3( and are superimposed here for comparison (dotted lines).

has a longer side chain in the substituting amino acid than F1625K and has a more pronounced shift in the first component of the inactivation curve but shows less acceleration of current decay. Despite their similar negative shift of the inactivation curve $\left(V_{\mathrm{h} 1}\right.$ or $V_{\mathrm{h}}$ of $-107.5,-122.7$, and $-116.5 \mathrm{mV}$ for $\mathrm{F} 1625 \mathrm{~K}$, F1625R, and F1625Q mutants, respectively) (Fig. 2A, 3A), the kinetics of development of inactivation as well as the kinetics of recovery from inactivation in the positive-charge mutants (especially F1625R) are evidently different from those in the F1625Q mutant. These findings may indicate that the existence of the new intermediate state caused by the added positive charge retards the kinetics of both further outward movement of S4/D4 (for the development of inactivation) and further inward movement of S4/D4 (for the recovery from inactivation), and arginine probably is more effective than lysine in doing so.

\section{Discussion}

\section{$\mathrm{Na}^{+}$channel inactivation is decisively controlled by the} movement of S4/D4

In this study, we replaced F1625 (the residue located just external to S4/D4) with different neutral and positive-charge amino acids and observed dramatic effects in channel inactivation. Because the recovery rates in the mutant and wild-type channels are different only at more positive potentials but all reach the same voltage-independent level at very negative potentials (Fig. 7A), the mutations do not seem to alter the final voltage-independent step in channel inactivation (e.g., the inactivation gate itself). What is altered is probably the upstream voltage-dependent step, namely the movement of related voltage sensors. Because F1625 is located just next to S4/D4, the externally located mutations very likely influence the movement of S4/D4, the transmembrane voltage sensor most implicated in inactivation (Stühmer et al., 1989; Chahine et al., 1994; Chen et al., 1996; Kühn and Greeff, 1999; Sheets et al., 1999). S4/D4 then faithfully transmits the allosteric effect from the mutation site to the internally located inactivation gate. In this regard, it is interesting that some mutant channels are inactivated at very negative potentials where there is hardly any channel activation (Fig. $2 A$ ). This finding would indicate that the altered S4/D4 movement itself could induce $\mathrm{Na}^{+}$ channel inactivation. In other words, the inactivation gate may be under the decisive control of S4/D4. We have also seen that neutral mutants of F1625 show a shift in the voltage axis with insignificant change in the steepness of the voltage dependence of different inactivation parameters (Figs. 3, 5). The neutral mutations thus probably stabilize the inactivated state without changing the basic voltage-dependent gating strategies of the channel. Moreover, the inactivation-stabilizing effect of the neutral mutations appears to be closely dependent on the physicochemical properties of the substituting side-chain group, especially its length and ability to form hydrogen bonds (Figs. 3, 4). As a first approximation, the additional stabilization energies on the inactivated state are roughly estimated by the product of the shift and the apparent equivalent gating charges in the inactivation curve and are $\sim 0.6, \sim 1.7, \sim 3.0$, and $\sim 5.7 \mathrm{kcal} / \mathrm{mol}$ for the $\mathrm{F} 1625 \mathrm{C},-\mathrm{N}$, $-\mathrm{H}$, and $-\mathrm{Q}$ mutants, respectively (Fig. $3 A$ ). These values are also consistent with the usual energy range of a hydrogen bond ( $1 \sim 5$ $\mathrm{kcal} / \mathrm{mol}$ ) (Zimmerman and Feldman, 1989). We therefore propose that the neutral mutations may cause new intramolecular interactions (most likely new hydrogen bonds) and thus stabilize S4/D4 in the "inactivation position." Altogether our data indicate that the outward movement of S4/D4 during depolarization is not a bystander phenomenon but has a decisive causal relation to $\mathrm{Na}^{+}$channel inactivation. When S4/D4 moves outward during depolarization (Chahine et al., 1994; Yang and Horn, 1995; Yang et al., 1996, 1997), its top is probably driven into an area with strong hydrogen bond-forming ability. The strength of hydrogen bonding may profoundly influence the tendency of S4/D4 to stay in this outward position and consequently profoundly influence channel inactivation.

\section{Electrostatic interactions are important in the determination of S4/D4 position}

The inactivation curves of positive-charge mutants of F1625 are split into two components (Fig. 2A). The first component suggests that a voltage-dependent inactivation process is manifest even at very negative potentials, but channel inactivation is not stable with this process and complete or stable inactivation would require the other process (the second component of the inactivation curve), which most likely happens after channel activation and is very much analogous to the inactivation in the wild-type and neutral mutant channels. Because the less stable inactivation state appears only in the positive-charge mutants but not in the wild-type or neutral mutant channels, some electrostatic force might be involved in the production of this state. The positive charge at 1625 , which is located slightly external to the constitutive positive charges in S4/D4, may have a chance to interact with the putative countercharges of S4/D4 (see below), leading to slight outward translocation of S4/D4. Because S4/D4 movement decisively controls the inactivation gate, the slight outward translocation of S4/D4 may result in an increased (but not stable) binding of the hinged lid to its receptor (i.e., new intermediate inactivation state in which the binding is not tight enough and significant unbinding happens in the steady-state condition). By the same token, the lack of initial delay in the course of recovery from inactivation is observed only in the positive-charge mutants. The positive charge at 1625 may have a chance to replace a constitutive S4/D4 positive charge in binding to the countercharges of S4/D4. This would enable a slight inward movement of the fully externally translocated S4/D4 and thus incomplete but 


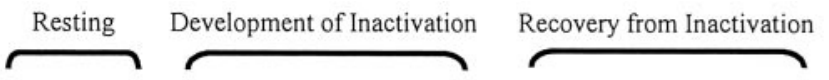

A. wild type :
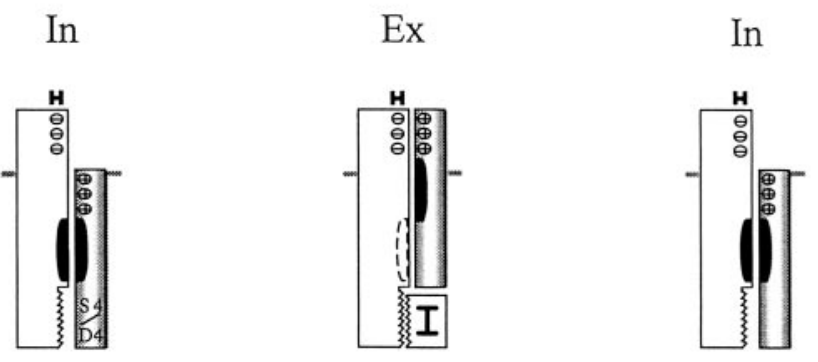

B. neutral mutants of F1625:

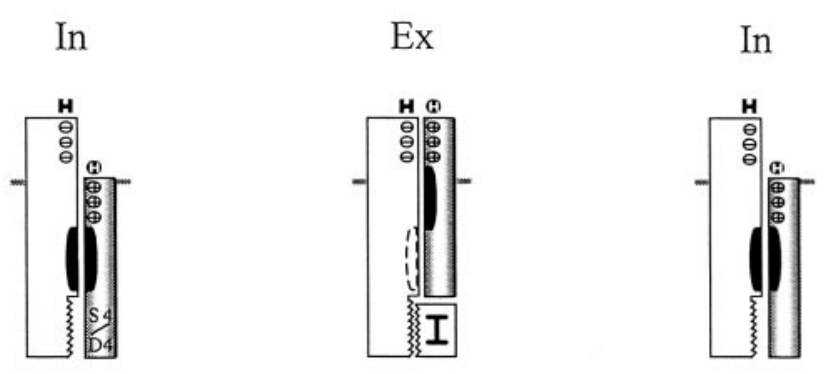

C. positive-charge mutants of F1625:
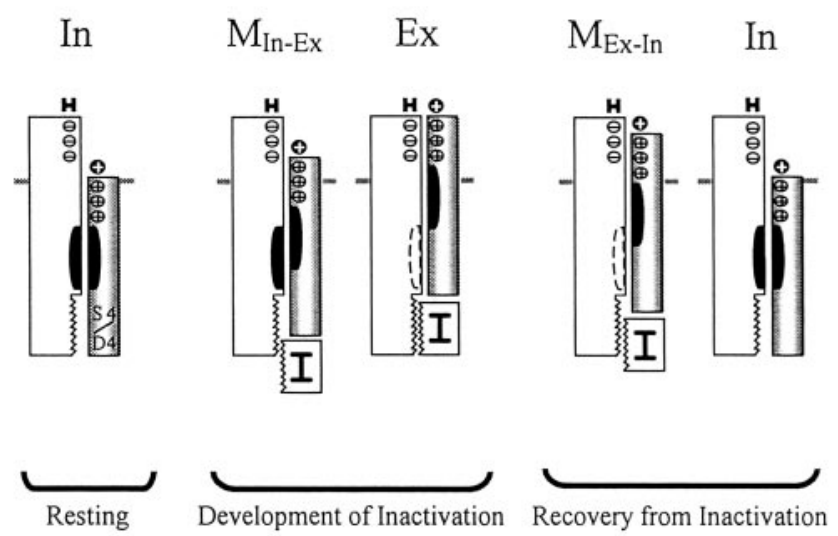

Figure 8. Schematic model describing the effect of $S 4 / D 4$ position on $\mathrm{Na}^{+}$channels inactivation. S4/D4 is shown as a shaded bar with the three outermost positive charges (R1, R2, and R3) near its top. The other part of the channel (presumably the gating canal of S4/D4) contains three discrete negative countercharges (CR1, CR2, and CR3, designating the countercharges for $\mathrm{R} 1, \mathrm{R} 2$, and $\mathrm{R} 3$ in the fully inactivated channel, respectively) and a hydrogen bonding area (symbolized by $\mathrm{H}$ ). The horizontal thin gray bars mark the external boundary of the cell membrane. The filled circles with the white $H$ and the white positive charge above the S4/D4 denote neutral and positive-charge substitutions of the $F 1625$ residue, respectively. In, Ex, and $M_{1 n-E x}$ or $M_{\mathrm{Ex}-\mathrm{In}}$ mean that S4/D4 is at the most internal, most external, and intermediate (when translocating from internal to external or from external to internal sides) positions, respectively. The one-side toothed square labeled with I and the opposite toothed surface comprise the inactivation gate (the hinged lid and its receptor). More external position of S4/D4 would allow larger contact area between the two toothed surfaces and consequently a more tightly bound hinged lid and a more stable inactivated state. For the sake of simplicity we illustrate the movement significant recovery from inactivation in the absence of channel deactivation (Fig. 6). These unique features of the positive-charge mutants strongly indicate that the regularly spaced positive charges in S4/D4 are "voltage sensors" not only for a long-range electric force (the membrane electric field), but also for a very localized shortrange electric force (the electric field of each specific counter negative charges). Functionally speaking, the latter (the specific electrostatic or charge-charge interaction) is also a decisive factor controlling the S4/D4 position and therefore the inactivation status of the $\mathrm{Na}^{+}$ channel. The important role of the short-range electric force may also provide functional insight into the electrostatic interaction between the outermost $\mathrm{S} 4$ charges and residue E418 in the activated $\mathrm{K}^{+}$channels (Elinder et al., 2001b).

\section{$\mathrm{Na}^{+}$channel inactivation is delicately controlled by the position of S4/D4}

It is interesting that the effects on channel inactivation of F1625K and F1625R are qualitatively similar but quantitatively very different (Figs. 2, 6, 7). The side chains of both $\mathrm{K}$ and $\mathrm{R}$ should point to the same space, but their positive charges are slightly different in shape (arginine has a larger and more plane space of charge distribution) and location (arginine has a longer side chain by $\sim 1.3 \AA$, the length of a resonating $\mathrm{C}=\mathrm{N}$ bond). If the slight geometrical and $1.3 \AA$ differences in charge distribution really matter, then the positive charge at 1625 probably interacts with one discrete countercharge at a time (i.e., a very specific shortrange force) rather than simultaneously with multiple surrounding negative charges. We therefore propose a model to explain our findings with discrete charge-charge interactions (Fig. 8). We have noted that F1625R has a smaller yet more negatively shifted first component of the inactivation curve than F1625K (Fig. 2) and thus a less externally translocated S4/D4 in F1625R in

$\leftarrow$

of S4/D4 as translational, but it can be easily modified to incorporate concomitant rotational movement with similar rationales. A, Left panel, In the resting wild-type channel, S4/D4 is restricted or stabilized at the resting (In) position by coupling to the deactivated conformation of the channel. The activation-inactivation coupling mechanism is represented by the (paired) black elliptical rectangles, larger contact area between which signals larger coupling energy to stabilize the particular protein conformation. Middle panel, Translocation of S4/D4 is triggered by channel activation, which makes significant conformational changes in one of the paired coupling rectangles (drawn in dashed lines and in white). S4/D4 is thus released from the In position and relocated to the normal inactivated (Ex) position via the electrostatic interactions at its top. Right panel, Channel deactivation resumes the favorable interaction between the coupling rectangles. Because sufficient energy is provided to compensate for the disrupted electrostatic interactions, S4/D4 is moved back to the In position. B, Neutral mutations of F1625 introduce new hydrogen bonds at the top of S4/D4 to further stabilize S4/D4 at the Ex position. The other basic gating strategies remain the same as those in the wild-type channel. C, Left panel, The resting state of the positive-charge mutant channels (F1625K and F1625R) is similar to that of the wild-type channel. Middle panel, The added positive charge on the top of S4/D4 could interact with the closest negative charge in the S4/D4 gating canal (presumably CR2 or (R3 rather than (R1) and induce a slight outward movement of S4/D4. This slight movement of S4/D4 is possible even in the absence of activation because the new electrostatic interaction could compensate for the distorted coupling between the black elliptical rectangles. However, the slightly outward intermediate S4/D4 position $\left(\mathrm{M}_{\mathrm{ln}-\mathrm{Ex}}\right)$ does not make a stable inactivated conformation, and the channel would still have a significant chance of being available (i.e., with an unbound hinged lid). Only when the channel is activated can S4/D4 move to the Ex position to make the same fully inactivated conformation as that in the wild-type and neutral mutant channels. Right panel, During repolarization, S4/D4 may move slightly inward from position Ex to $M_{E x-I n}$, even before channel deactivation, and thus no initial delay is found in the recovery courses of the channels from inactivation. This movement is possible in the positive-charge mutants because of the new electrostatic interaction between the added positive charge and the closest negative charge (presumably (R1). $\mathrm{Na}^{+}$channel deactivation resumes favorable interaction between the rectangles and therefore a further inward movement of S4/D4 back to the In position, which is responsible for the second (the more accelerated and more complete) recovery phase from inactivation. 
the intermediate inactivated state originating from the resting channel (Fig. $8 C, \mathrm{M}_{\text {In-Ex }}$ ). This is as if $\mathrm{F} 1625 \mathrm{~K}$ requires a small (presumably $\sim 1.3 \AA$ ) movement of the main peptide chain to compensate for the difference in side chain (lengths and other physicochemical properties) to attain a similar interaction with the negative countercharge. The $\sim 1.3 \AA$ movement does not seem to be trivial in the absence of channel activation and would require $\sim 15 \mathrm{mV}$ (the difference between $V_{\mathrm{h} 1}$ of F1625R and F1625K mutants) (Fig. $2 \mathrm{~A}$ ) additional change in the membrane potential to accomplish. The faster initial recovery phase in F1625K than in F1625R (Fig. 6) can also be envisaged in the same way. There may be slightly different movements of S4/D4 between F1625K and F1625R for the $\mathrm{M}_{\mathrm{Ex} \text {-In }}$ position (Fig. 8C), leading to a less stabilized inactivation gate and a faster initial recovery rate in F1625K. The control of the inactivation gate by S4/D4 position thus might be so delicate that a small $(\sim 1.3 \AA)$ S4/D4 movement could significantly alter the inactivation status.

\section{Implications regarding the functional design and gating conformational changes of the $\mathrm{Na}^{+}$channel}

It has been shown that R1626, R1629, and R1632 (R1, R2, and R3) residues in S4/D4 move outside the membrane during depolarization (Chahine et al., 1994; Yang and Horn, 1995; Yang et al., 1996). As a first approximation, there may be three corresponding negative charges, namely CR1, CR2, and CR3 (the countercharges responsible for binding to R1, R2, and R3 in the fully inactivated channel, respectively) (Fig. 8) along the path of S4/D4 movement or the "S4/D4 gating canal." If the top part of S4/D4 (including residues 1625-1632) has an $\alpha$-helical structure in the resting channels, then our findings would indicate that S4/D4 must have a translational movement along the axis of the $\alpha$-helix during channel activation-inactivation, no matter with which countercharge residue $1625 \mathrm{R}$ interacts. Even if $1625 \mathrm{R}$ interacts with CR1 to make the intermediate inactivated state in Figure $2 \mathrm{~A}$ $\left(\mathrm{M}_{\text {In-Ex }}\right.$ in Fig. $\left.8 C\right)$, the S4/D4 helix should have an additional translational displacement of $1.5 \AA$ during channel activationinactivation so that R1 or R1626 is moved to CR1 in the fully inactivated channel. If $1625 \mathrm{R}$ interacts with CR2 or CR3 instead of CR1 to make the intermediate position $\mathrm{M}_{\mathrm{In}-\mathrm{Ex}}$, then the additional axial translation would be 6 or $10.5 \AA$. The gating conformational change of S4/D4 thus cannot be purely rotational but at least like a "helical screw" (Catterall, 1986; Guy and Seetharamulu, 1986; Lecar and Larsson, 1997). On the other hand, the decisive and delicate control of the inactivation gate by S4/D4 suggests that the activation-inactivation coupling very likely involves S4/D4 (Fig. 8, black elliptical rectangles). However, because there is no corresponding change in the voltage dependence of activation in the F1625 mutants (Figs. $2 \mathrm{~B}, 3 \mathrm{~B}$ ), the movement of S4/D4 probably happens well after the major events leading to channel activation. S4/D4 thus could be a key apparatus in the activation-inactivation coupling but should be located very close to the inactivation end of the whole coupling mechanism. In view of its decisive and delicate controlling effect, S4/D4 may be located so close to the inactivation end that it has a direct physical connection with the inactivation gate. Because the four peptide domains (with their respective $\mathrm{S} 4$ segments) in the $\mathrm{Na}^{+}$ channel are homologous structures and similar motifs are also found in the $\mathrm{K}^{+}$and $\mathrm{Ca}^{2+}$ channels, it would be interesting to determine whether the direct connection between the voltage sensor and the corresponding gate is a more general design applicable to the S4(-like) voltage sensors in the other domains of the $\mathrm{Na}^{+}$channel and in the other voltage-gated ion channels.

\section{References}

Armstrong CM, Bezanilla F (1977) Inactivation of the sodium channel. II. Gating current experiments. J Gen Physiol 70:567-590.

Auld VJ, Goldin AL, Krafte DS, Catterall WA, Laster HA, Davidson N, Dunn RJ (1990) A neutral amino acid charge in segment IIS4 dramatically alters the gating properties of the voltage-dependent sodium channel. Proc Natl Acad Sci USA 87:323-327.

Bezanilla F, Armstrong CM (1977) Inactivation of the sodium channel. I. Sodium current experiments. J Gen Physiol 70:549-566.

Catterall WA (1986) Molecular properties of voltage-sensing sodium channels. Annu Rev Biochem 55:953-985.

Chahine M, George Jr AL, Zhou M, Ji S, Sun W, Barchi RL, Horn R (1994) Sodium channel mutations in paramyotonia congenita uncouple inactivation from activation. Neuron 12:281-294.

Chen L-Q, Santarelli V, Horn R, Kallen RG (1996) A unique role for the S4 segment of domain 4 in the inactivation of sodium channels. J Gen Physiol 108:549-556.

Elinder F, Århem P (1999) Role of the individual surface charges of voltagegated K channels. Biophys J 77:1358-1362.

Elinder F, Århem P, Larsson HP (2001a) Localization of the extracellular end of the voltage sensor S4 in a potassium channel. Biophys J 80:1802-1809.

Elinder F, Mannikko R, Larsson HP (2001b) S4 charges move close to residues in the pore domain during activation in a $\mathrm{K}^{+}$channel. J Gen Physiol 118:1-10.

Fleig A, Fitch JM, Goldin AL, Rayner MD, Starkus JG, Ruben PC (1994) Point mutations in IIS4 alter activation and inactivation of rat brain type IIA Na channels in Xenopus oocyte macropatchs. Pflügers Arch 427:406-413.

Goldman L, Kenyon JL (1982) Delays in inactivation development and activation kinetics in Myxicola giant axons. J Gen Physiol 80:83-102.

Goldman L, Schauf CL (1972) Inactivation of the sodium current in Myxicola giant axons: evidence for coupling to the activation process. J Gen Physiol 59:659-675.

Guy HR, Seetharamulu P (1986) Molecular model of the action potential sodium channel. Proc Natl Acad Sci USA 83:508-512.

Horn R (2000) A new twist in the saga of charge movement in voltagedependent ion channels. Neuron 25:511-514.

Kontis KJ, Rounaghi A, Goldin AL (1997) Sodium channel activation gating is affected by substitutions of voltage sensor positive charges in all four domains. J Gen Physiol 110:391-401.

Kühn FJP, Greeff NG (1999) Movement of voltage sensor S4 in domain 4 is tightly coupled to sodium channel fast inactivation and gating charge immobilization. J Gen Physiol 114:167-183.

Kuo C-C, Bean BP (1994) $\mathrm{Na}^{+}$channels must deactivate to recover from inactivation. Neuron 12:819-829.

Kuo C-C, Yang S (2001) Recovery from inactivation of T-type $\mathrm{Ca}^{2+}$ channels in rat thalamic neurons. J Neurosci 21:1884-1892.

Larsson HP, Elinder F (2000) A conserved glutamate is important for slow inactivation in $\mathrm{K}^{+}$channels. Neuron 27:573-583.

Lecar H, Larsson HP (1997) Theory of S4 motion in voltage-gated channels. Biophys J 72:341a.

Patton DE, Isom LL, Catterall WA, Goldin AL (1994) The adult rat brain $\beta 1$ subunit modifies activation and inactivation gating of multiple sodium channel $\alpha$ subunit. J Biol Chem 269:17649-17655.

Qu Y, Rogers JC, Chen S-F, McCormick KA, Scheuer T, Catterall WA (1999) Functional roles of the extracellular segments of the sodium channel $\alpha$ subunit in voltage-dependent gating and modulation by $\beta 1$ subunits. J Biol Chem 274:32647-32654.

Sheets MF, Kyle JW, Kallen RG, Hanck DA (1999) The Na channel voltage sensor associated with inactivation is localized to the external charged residues of domain IV, S4. Biophys J 77:747-757.

Stühmer W, Conti F, Suzuki H, Wang XD, Noda M, Yahagi N, Kubo H, Numa S (1989) Structural parts involved in activation and inactivation of the sodium channel. Nature 339:597-603.

Yang N, Horn R (1995) Evidence for voltage-dependent S4 movement in sodium channels. Neuron 15:213-218.

Yang N, George Jr AL, Horn R (1996) Molecular basis of charge movement in voltage-gated sodium channels. Neuron 16:113-122.

Yang N, George Jr AL, Horn R (1997) Probing the outer vestibule of a sodium channel voltage sensor. Biophys J 73:2260-2268.

Zimmerman JJ, Feldman S (1989) Physical-chemical properties and biological activity. In: Principles of medicinal chemistry, Ed 3 (Foye WO, ed), pp 7-37. Malvern, PA: Lea \& Febiger. 